\title{
Article \\ Variable Repetition Rate Picosecond Master Oscillator for Photoelectron Gun
}

\author{
Mikhail V. Gorbunkov ${ }^{1}\left(\mathbb{D}\right.$, Yulia Y. Maslova ${ }^{1, *} \mathbb{(}$, Yurii V. Shabalin ${ }^{1}(\mathbb{D})$ and Vladimir G. Tunkin ${ }^{2}$ \\ 1 Department of Quantum Radiophysics, P.N. Lebedev Physics Insitute, Leninskii pr. 53, \\ 119991 Moscow, Russia; gorbunkovmv@lebedev.ru (M.V.G.); shabal@yandex.ru (Y.V.S.) \\ 2 International Laser Center, Lomonosov Moscow State University, Leninskie Gori 1, 119991 Moscow, Russia; \\ vladimirtunkin@mail.ru \\ * Correspondence: jmaslova@mail.ru
}

\section{check for} updates

Citation: Gorbunkov, M.V.; Maslova, Y.Y.; Shabalin, Y.V.; Tunkin, V.G. Variable Repetition Rate Picosecond Master Oscillator for Photoelectron Gun. Photonics 2022, 9, 106. https:// doi.org/10.3390/photonics9020106

Received: 31 December 2021 Accepted: 10 February 2022 Published: 13 February 2022

Publisher's Note: MDPI stays neutral with regard to jurisdictional claims in published maps and institutional affiliations.

Copyright: (C) 2022 by the authors. Licensee MDPI, Basel, Switzerland. This article is an open access article distributed under the terms and conditions of the Creative Commons Attribution (CC BY) license (https:// creativecommons.org/licenses/by/ $4.0 /)$.

\begin{abstract}
In this work, aiming at the master oscillator of the photoelectron gun with a variable repetition rate of electron bunches, a picosecond solid-state laser subject to delayed optoelectronic feedback and RF loss modulation is studied. Loss modulation is performed using an electro-optical modulator with zero bias at the second accelerator frequency subharmonic. Optoelectronic negative feedback uses an intracavity electro-optical modulator and a fast high-voltage photodiode mounted as close as possible to the modulator crystal. An analytical formula is obtained for the pulse duration, and estimates are given for $\mathrm{Nd}$ and $\mathrm{Yb}$ based media and $\mathrm{L}, \mathrm{S}, \mathrm{C}$ and $\mathrm{X}$-band used in modern linear accelerators. Numerical simulation proves that the control is suitable for pulse-repetitive operation. The proposed approach solves the problem of laser pulse shortening and locking the master oscillator, and therefore, electron bunches in photoelectron guns, to the high-stable RF generator controlling accelerator functioning.
\end{abstract}

Keywords: solid state picosecond lasers; negative optoelectronic feedback; electrooptic modulation; photoinjectors

\section{Introduction}

In many fields of science and technology, trains of picosecond pulses play an important role. One can mention, e.g., the following technological applications: ultrafast laser-material and tissue processing [1], telecommunication [2], optical clocking [3], etc. As to the trains consisting of a finite number of picosecond pulses, one should note the problems arising in electron accelerator technology, i.e., the development of a photoelectron gun for multibunch linear accelerators $[4,5]$. Such photoelectron guns play an important role in bright $\mathrm{X}$-ray sources, namely FELs and Thomson generators [6]. Laser pulses repetition rate should match the frequency of linear accelerator, and the photoelectron gun laser pulses should coincide with selected maxima of the resonator's microwave field in $\mathrm{GHz}$ range: 1-2 GHz in L-band, 2-4 GHz in S-band [7], 4-8 GHz in C-band, or 8-12 GHz in X-band. In Thomson sources, picosecond pulses are also used for interaction with electron bunches [8].

For next-generation sources, the minimization of the photoinjector electron bunch emittance is of great importance [9]. In best photoelectron guns designed for bunch charge of $1 \mathrm{nC}$, the emittance is as low as $1 \mathrm{~mm} \cdot \mathrm{mrad}$ [10]. The solution is to reduce the individual bunch charge and at the same time to increase the number of bunches in the train by increasing their repetition rate. For a low-emittance (low-charge) photoelectron gun, the required laser pulse energy is about $10-100 \mathrm{~nJ}$ at $1 \mu \mathrm{m}$ wavelength.

As to picosecond systems with variable repetition rate $\geq 100 \mathrm{MHz}$ up to $1 \mathrm{GHz}$, two works can be mentioned [11,12]. In both of them, the optical parametric oscillator was synchronously pumped by picosecond pulses of the gain-switched laser diode amplified by a chain of Yb-doped [11] or Tm-doped [12] fiber amplifiers. One or an integer number of pulses circulated in the optical parametric oscillator cavity simply by controlling the 
gain-switched laser diode repetition rate without readjusting the parametric oscillator cavity. The laser system [11] is the closest to fulfilling the requirement of pulse energy up to 10-100 nJ. It consists of a gain-switched laser diode and a chain of diode-pumped $\mathrm{Yb}$ doped fiber amplifiers. It delivers $21 \mathrm{ps}$ pulses with average power up to $100 \mathrm{~W}$ at a center wavelength of $1.06 \mu \mathrm{m}$. The repetition rate can be varied between $100 \mathrm{MHz}$ and $1 \mathrm{GHz}$ through the use of an electro-optical modulator pulse-picker. The system did not provide the required duration of less than $5 \mathrm{ps}$, and reaching several ps duration would require a stretcher-compressor technique, which would further complicate the device. Moreover, when using the quasi-CW laser system to generate electron bunches in a photoinjector, only a smaller part of the laser pulses would be used.

Aiming at the pulse-repetitive regime suitable for muli-bunch photo-electron guns, we propose a bulk solid-state laser with pulsed diode pumping and negative feedback control. The development of powerful and stable picosecond sources of laser pulse trains with pulse repetition rates higher than the reciprocal cavity round-trip time $T_{r}$ requires the development of new approaches in radiation temporal structure organization that would provide powerful short pulses with controllable timing for synchronization in $\mathrm{GHz}$ frequency range. This range is far from the fundamental frequency of powerful solid-state lasers but convenient to match up directly to the RF clocks. The solution to this problem proposed in this paper is based on harmonic mode-locking (HML) when not one but several pulses circulate in a laser cavity. The HML term was introduced in the mid-60s by the authors who investigated the process of generating several pulses in the cavity during active mode-locking using an intracavity phase modulator [13]. HML enhances the output pulses repetition rate from the fundamental frequency $\left(f_{c}\right)$ with a typical level of $100 \mathrm{MHz}$ up to the level of 1-20 GHz by generating higher harmonics, higher than 200th harmonics in some papers [14].

Different HML methods were elaborated up to now in fibre lasers. In [15,16], soliton passive HML was obtained in an Er-doped fibre ring laser. In [17], HML was achieved by dissipative solitons production also in Er-doped fibre laser. Active HML was demonstrated in undoped fibre laser based on semiconductor optical amplifier [18] and the same operational mode was realized in Er-doped fibre laser [19]. In both papers, $\mathrm{LiNbO}_{3}$ electrooptic modulator was used, and laser pulses repetition rate was changed by varying the modulator working frequency at fixed pump power. The highest 928th harmonics of $f_{c}$ corresponding to a frequency of $22.2 \mathrm{GHz}$ was achieved in [15]. HML pulses energy obtained in above-mentioned papers was at the level of 1-10 pJ for soliton operational mode and hundreds of $\mathrm{pJ}$ for dissipative operational mode.

Much higher energy pulses needed for photoelectron gun operation (10-100 nJ at the wavelength of $1 \mu \mathrm{m}$ ) can be achieved using solid-state bulk lasers pumped by an array of laser diodes [20]. A frequently occurring method of achieving HML in such types of lasers is the implementation of electro-optic modulation [21-23]. Electro-optic phase modulation based on $\mathrm{LiNbO}_{3}$ crystal was successfully used in $\mathrm{Nd}$ :YAG laser to obtain quite short picosecond pulses at the fundamental frequency of $235 \mathrm{MHz}$ [24]. To obtain higher repetition rates, $\mathrm{LiNbO}_{3}$ crystal was placed in a resonant microwave cavity. In this way, $22 \mathrm{~d}$ harmonics $(1.76 \mathrm{GHz})$ was achieved in [21], 84th harmonics $(19.97 \mathrm{GHz})$ was obtained in [22].

HML mechanism essentially different from those given above was experimentally realized in [25]. Up to 4th harmonics was obtained in Nd:YLF laser pumped by laser diode array with the power of $70 \mathrm{~W}$. HML was achieved using intracavity Pockels-cell governed by fast optoelectronic negative feedback (NFB) with adjustable delay. Later the concept of the self-starting harmonic mode-locking (SSHML) by electro-optical feedback in a solid-state laser was studied in [26]. Such an operational mode with $m$ equidistant pulses in a laser cavity is controlled by a delayed single negative electro-optical feedback. It was shown that the analytical consideration of the steady-state generation and the numerical simulation of SSHML dynamics starting from the spontaneous emission noise level provide the complete description essential for applications. 
We have also previously proved the unique ability of the electro-optical negative feedback circuit to select a single pulse on the axial interval synchronized with an external high-frequency harmonic signal [27]. In this case, in addition to synchronization, the modulator makes it possible to significantly reduce the pulse duration, to make it close to optimal for photoelectron injector.

In this paper, a new design of a picosecond laser is proposed for a photoelectron gun of a multibunch linear accelerator. It is based on the idea to combine optoelectronic negative feedback HML and RF amplitude modulation (L, S, C and X-band used in modern linear accelerators). The master oscillator generates a desired number (from 2 to more than 10) of synchronized pulses during one round trip of the laser cavity with the total number of up to $10^{6}$ in the train. The proposed solution will advance the development of the new generation of $X$-ray sources, i.e., laser-electron $X$-ray generators and FELs, by reducing the emittance of the photoelectron gun down to $0.2 \mathrm{~mm} \cdot \mathrm{mrad}$.

\section{Solid-State Laser with Electro-Optical Control and RF Modulation}

The scheme of the proposed master oscillator is shown in Figure 1. Two highly reflective mirrors M1 and M2 form the laser resonator. Negative feedback employs an intracavity electro-optical modulator $\mathrm{EOM}_{1}$ which includes a polarizer, an electro-optical crystal, and an optoelectronic control RC-circuit. The current source of the circuit is a fast high-voltage photodiode attached directly to the electro-optical crystal without additional amplification, which is the unique feature of our approach. The capacity $C$ of the control circuit is the capacity of the electro-optical crystal. A discharge resistor $R$ controls the time constant $\tau=R C$ of the feedback circuit (see Figure 2). An intracavity beam-splitter is installed between $\mathrm{EOM}_{1}$ and the laser active medium. The reflection from the beam-splitter is utilized in the feedback loop, while another reflection is the laser output. If the feedback loop utilizes the reflection from the $\mathrm{EOM}_{1}$ polarizer, then the stability region narrows significantly [28], which means a much lower operational gain level. An aperture selects the fundamental mode.

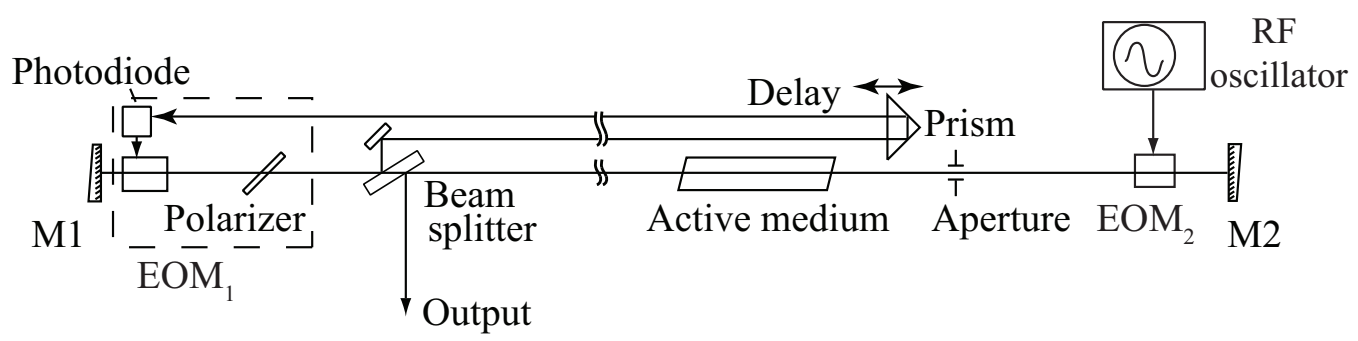

Figure 1. The simplified scheme of a master picosecond oscillator: a delayed-feedback-controlled harmonically mode-locked laser with external RF modulation. M1, M2 are resonator mirrors; $\mathrm{EOM}_{1}$ is electro-optical modulator for SSHML, $\mathrm{EOM}_{2}$ is the electro-optical modulator for synchronization, delay $T_{d}$ is set to $\simeq(1-1 / m) T_{r}$ by shifting the prism.

The second RF-driven electro-optical modulator $\mathrm{EOM}_{2}$ is installed at a distance from mirror M2 that is a multiple of half the modulation period. The SSHML operation occurs in some separate intervals on the delay scale. In the experiment [25], the delay time $T_{d_{L}}$ was adjusted to obtain the regular train of picosecond pulses on the oscilloscope up to 4 pulses per cavity round-trip time by moving the delay line prism. For this purpose, this prism was mounted on a micrometric translation stage, which in turn was mounted on an optical rail to move into these separate delay intervals. 
a

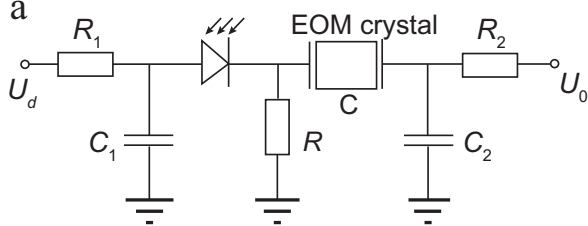

b

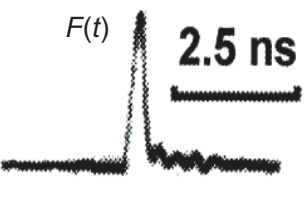

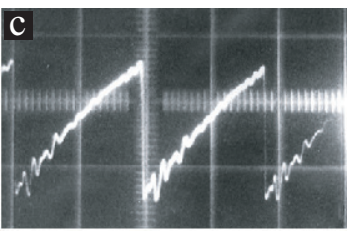

Figure 2. Electro-optical control circuit and signals: (a) equivalent circuit, $U_{0}$ is $\mathrm{EOM}_{1}$ bias voltage, $U_{d}$ is photodiode bias voltage, $R$ is a discharge resistor; ballast resistors $R_{1}$ and $R_{2}$ connect the voltage sources $U_{d}$ and $U_{0}$ to the circuit, $C$ is EOM $E_{1}$ capacity, $C_{1}$ and $C_{2}$ are capacitors such that $C_{1} \gg C_{2}$, $C_{2}>C$; (b) photocurrent pulse; (c) $\mathrm{EOM}_{1}$ control voltage, horizontal scale is $5 \mathrm{~ns} /$ div, vertical scale is $1 \mathrm{~V} /$ div which corresponds to the modulator control voltage of $250 \mathrm{~V} / \mathrm{div}$.

Let us consider the steady SSHML regime with $m$ equal and equidistant pulses in the cavity (Figure 3). It is quite similar to the case of the self-mode-locking with a single pulse. We denote by $T_{d}$ the delay between the laser pulse intensity maximum and the maximum of the photocurrent that is generated by the part of the same pulse deflected into the feedback loop. Each of the pulses passes through the $\mathrm{EOM}_{1}$ close to the moment of its maximum transmission. At this moment, $C$ discharges to a minimal voltage. Below we refer to the case of exact match of the pulse passage and the maximum transmission as the maximum transmission case. Since the discharge duration is limited to the time $T_{r} / m$, which is interpulse interval, we obtain that $T_{d_{L}}=(1-1 / m) T_{r}+\Delta T$, where $\Delta T$ is the time between the maximum $\mathrm{EOM}_{1}$ transmission and the maximum of the photocurrent pulse. To keep the $\mathrm{EOM}_{1}$ transmission modulation depth constant, one should decrease the value of $\tau=R C$ inversely proportional to $m$. The maximum number of pulses $m$ is limited by the photodiode response time $\tau_{F}\left(\tau_{F}\right.$ is the FWHM of the photodiode time response function, see formula (2)). The multiple of $T_{r}$ added to the feedback delay retains the conditions for a certain operational mode.
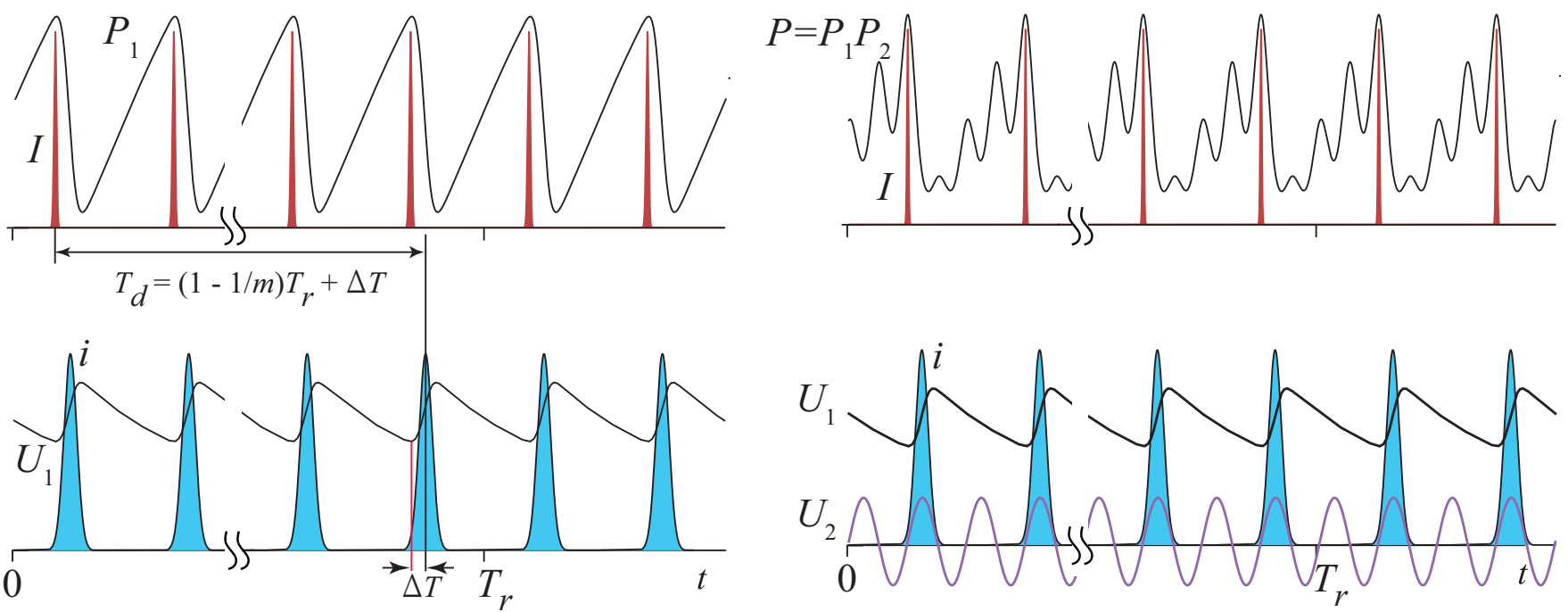

Figure 3. SSHML operational mode with $m$ pulses in the cavity: single NFB action (left) and combined action of NFB and RF modulation. Temporal profiles of laser intensity $I(t)$, photocurrent $i(t), \mathrm{EOM}_{1}$ control voltage $U_{1}(t), \mathrm{EOM}_{1}$ transmission $P_{1}(t), \mathrm{EOM}_{2}$ control voltage $U_{2}(t)$, total transmission $P(t)$. $T_{d}$ is the feedback delay, $T_{r}$ is the cavity round-trip time.

$\mathrm{EOM}_{2}$ is used to synchronize the master oscillator to the RF clock of the accelerator system of the photoelectron injector. It operates at zero bias and is driven by a subharmonic of the accelerating frequency. The transmission of $\mathrm{EOM}_{2}$ has several maxima at the time interval $T_{r} / m$. Each maximum corresponds to a zero of the periodic accelerating field of the photoelectron gun. Compared to SSHML using a single $\mathrm{EOM}_{1}$, the combined action of two modulators provides more flexible control over the output radiation parameters. It allows 
synchronizing laser pulses with the accelerator clock, controlling the pulse repetition rate and duration.

\section{Numerical Model}

Based on the approach proposed in [29], let us trace the evolution of the laser radiation during a round-trip along the cavity.

\subsection{Loss and Shaping in EOMs}

\subsection{1. $\mathrm{EOM}_{1}$ for $S S H M L$}

Photocurrent $i_{d}(t)$ in $\mathrm{EOM}_{1}$ control circuit is calculated as the convolution of the laser intensity $I(t)$ with the photocurrent response function $F(t)$ taking into account feedback loop sensitivity $k$ and optical delay $T_{d}$

$$
i_{d}(t)=k \int_{0}^{+\infty} I\left(t-t^{\prime}-T_{d}\right) F\left(t^{\prime}\right) d t^{\prime}
$$

For estimation, it is convenient to assume that $F(t)$ is Gaussian, i.e.,

$$
F(t)=\exp \left(-4 \ln 2 t^{2} / \tau_{F}^{2}\right) .
$$

Laser radiation intensity is

$$
I(t)=A(t) A^{*}(t),
$$

where $A^{*}(t)$ is complex conjugate of the amplitude $A(t)$. Expression (3) describes the unit system we are working in.

Since the control circuit includes the photodiode as a current source, the control voltage $U(t)$ applied to the electro-optic crystal is calculated from

$$
\frac{d U(t)}{d t}+\frac{U(t)}{R C}=\frac{i_{d}(t)}{C} .
$$

Then $\mathrm{EOM}_{1}$ transmission is

$$
P_{1}(t)=\cos ^{2}\left(\frac{\pi}{2} \cdot \frac{U(t)+U_{0}}{U_{\lambda / 4}}\right),
$$

where $U_{0}$ is the static bias voltage and $U_{\lambda / 4}$ is the Pockels cell quarter-wave voltage. If the control is designed according to the principle of negative feedback, then $P_{1}(t)$ decreases with $U(t)$ and with $I(t)$.

\subsection{2. $\mathrm{EOM}_{2}$ for Synchronization with Accelerator}

In the case of $\mathrm{EOM}_{2}$ controlled by RF oscillator, the transmission is

$$
P_{2}(t)=\cos ^{2}\left(\frac{\pi}{2}\left(\frac{U_{m}}{U_{\lambda / 4}} \cdot \sin \left(\frac{2 \pi}{T} t\right)+\frac{U_{m 0}}{U_{\lambda / 4}}\right)\right),
$$

where $U_{m}$ is the amplitude of harmonic voltage and $U_{\lambda / 4}$ is the Pockels cell quarter-wave voltage, $T$ is the period of RF oscillator signal. If $a=\frac{U_{m}}{U_{\lambda / 4}}<200 \%$, bias voltage $U_{m 0}=0$ and $T=2 T_{r} / k$, then the transmission of the modulator has $k$ equidistant maxima (equal to 1 ) per resonator round-trip time $T_{r}$. Figure 4 shows the modulator transmission $P_{2}(t)$ at $a=50,100,150,200 \%, k=2$ and two values of $U_{m 0} / U_{\lambda / 4}$ for comparison. In the case of nonzero bias voltage, maxima of $P_{2}(t)$ shift and are not equidistant. If $U_{m 0}=0$, the transmission modulation is periodic at double frequency of external RF oscillator. 

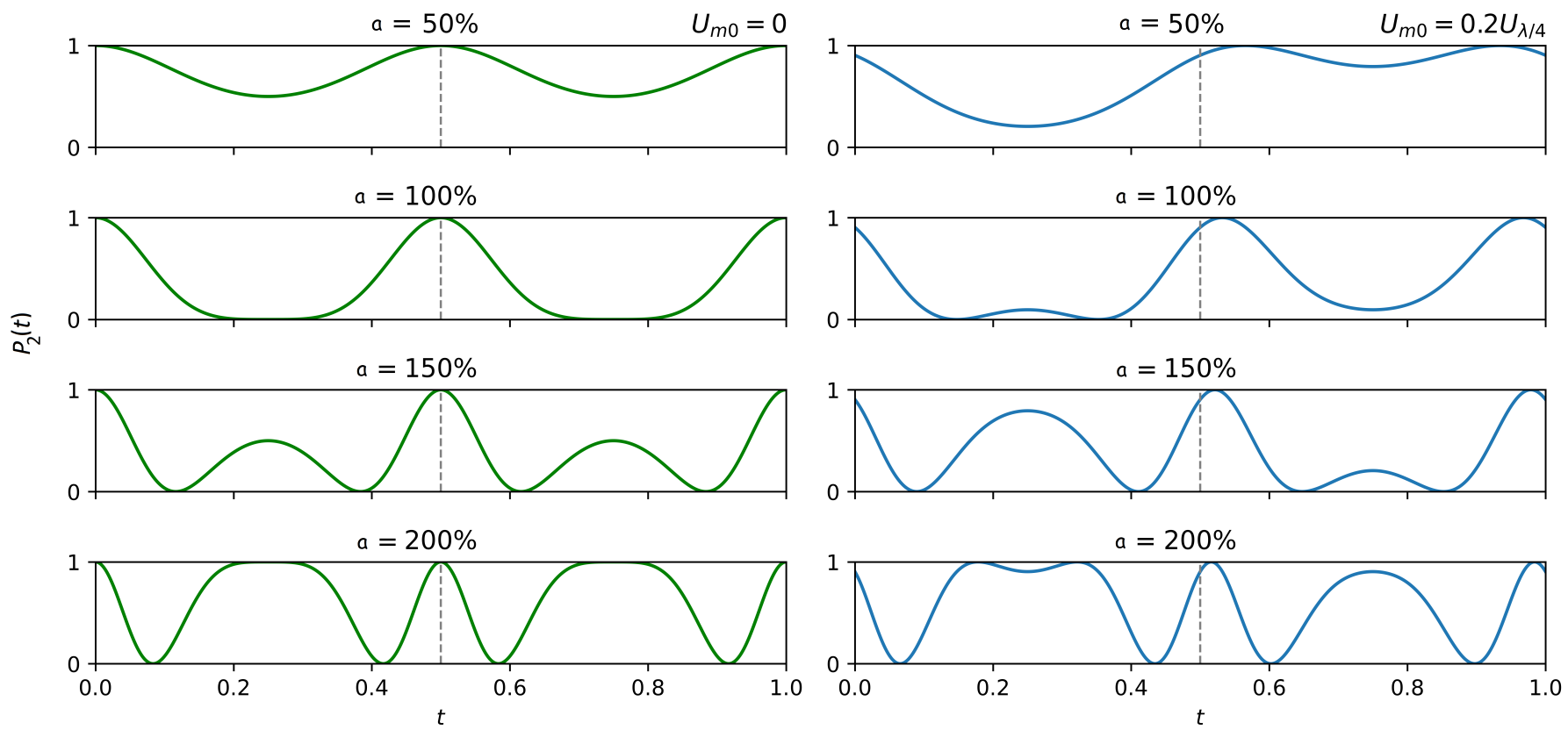

Figure 4. Transmission $P_{2}(t)$ for bias voltage $U_{m 0} / U_{\lambda / 4}=0$ (left) and 0.2 (right) at $a=$ $50,100,150,200 \%, k=2$. Time $t$ is in units of the resonator round-trip time $T_{r}$ (color online).

\subsection{Amplification in the Active Medium}

The radiation that passed the polarizer of $\mathrm{EOM}_{1}$ and the beam-splitter is further amplified twice in the active medium at $T_{r}$ (on the way to the mirror M2 and back) with (amplitude) gain coefficient $g$ at the central wavelength. We consider the feedback to be sufficiently sensitive so that the gain saturation is low. To describe the laser radiation passage through the active medium, we follow the approach of [30], which is also valid for high gain levels. The relation between outcoming radiation amplitude $A^{\text {out }}(t)$ and incoming radiation amplitude $A^{\text {in }}(t)$ after two passes through the active medium is:

$$
A^{\text {out }}(t)=\frac{\exp \left(\frac{3}{4} g\right)}{2 \sqrt{\pi} \tau_{a} \sqrt{g}} \cdot \int_{-\infty}^{\infty} A^{\text {in }}\left(t^{\prime}\right) \exp \left(\frac{t-t^{\prime}}{2 \tau_{a}}-\frac{\left(t-t^{\prime}\right)^{2}}{4 g \tau_{a}^{2}}\right) d t^{\prime},
$$

where $\tau_{a}=\left(\pi \Delta v_{L}\right)^{-1}, \Delta v_{L}$ is the active medium gain linewidth.

\subsection{Spontaneous Emission of the Active Medium}

At each round-trip, spontaneous emission noise $N(t)$ adds up to the laser radiation field. We assume that the longitudinal diode pumping focuses on the fundamental mode volume with waist $w$. Then the total average noise intensity is

$$
I_{\text {noise }}=h v \frac{g \lambda^{2}}{\pi^{2} w^{2} \sigma T_{a}},
$$

where $h v$ is the photon energy, $\lambda$ is the radiation wavelength, $\sigma$ is the amplification crosssection, $T_{a}$ is the upper state lifetime.

\subsection{Passive Loss}

Assuming that the beam splitter reflection coefficient is $S$, a linear decrease in intensity during each round-trip is described by a factor of $(1-S)^{2}$. The output radiation reflects from the beam-splitter after two passages through the active medium. 


\subsection{Final Form for Amplitude Transformation}

By collecting all terms that describe the radiation evolution, we can write the final form of amplitude transformation from round-trip $K$ to round-trip $(K+1)$ as

$$
A_{K+1}(t)=(1-S) \sqrt{P(t)} \frac{\exp g}{2 \sqrt{\pi} \tau_{a} \sqrt{g}} \times \int_{-\infty}^{\infty} A_{K}\left(t^{\prime}\right) \exp \left[-\left(\frac{t-t^{\prime}-\tau_{a} g}{2 \tau_{a} \sqrt{g}}\right)^{2}\right] d t^{\prime}+N_{K}(t),
$$

where

$$
P(t)=P_{1}(t) P_{2}(t) \text {. }
$$

The convolution term in expression (9) determines the laser pulse elongation, while the shape of $P(t)$ in the vicinity of its maximum determines the pulse shortening. By shifting the feedback delay, the behavior of $P(t)$ at the time interval of $T_{r}$ can be adjusted towards the appearance of several maxima and thus for the generation of several laser pulses per $T_{r}$.

\section{Steady-State Analysis}

The approximation of $P(t), A_{K}$ and $A_{K+1}$ by Gaussian functions [31] allows to find the solution of Equation (9) by direct substitution. We obtain the laser pulse duration under the combined action of NFB and RF modulation:

$$
\tau_{L M}=\sqrt{\frac{8 \ln 2}{\pi \Delta v}} \sqrt[4]{\frac{-g P_{\max }}{P^{\prime \prime}+P_{\max } P_{2}^{\prime \prime}}}
$$

Using the laser pulse duration $\tau_{L}$ in the maximum transmission case without modulation (as derived in [26]):

$$
\begin{gathered}
\tau_{L}=\left(\frac{2 \sqrt{\ln 2}}{\pi}\right)^{3 / 4} \frac{\left(\tau_{F} \cdot R C\right)^{1 / 4}}{\Delta v_{L}^{1 / 2}}\left(\ln \frac{G}{P_{0}(1-S)^{2}}\right)^{\frac{1}{4}} . \\
\cdot\left[\left(\frac{2}{\pi} \arccos \sqrt{\frac{P_{0}}{G}}-\frac{U_{0}}{U_{\lambda / 4}}\right) \sqrt{\frac{G}{P_{0}}-1}\right]^{-\frac{1}{4}}\left[\ln \left(\frac{2 \sqrt{\ln 2}}{\sqrt{\pi}}\left(\exp \frac{T_{r}}{m R C}-1\right) \frac{R C}{\tau_{F}}\right)\right]^{-\frac{1}{8}}
\end{gathered}
$$

and modulation-determined duration (see Appendix A)

$$
\tau_{M}=\frac{2}{\pi} \sqrt{\frac{\ln 2}{\pi}}\left(\frac{T}{\Delta v} \cdot \frac{U_{\lambda / 4}}{U_{m}}\right)^{1 / 2}\left(\ln \frac{G}{P_{0}(1-S)^{2}}\right)^{1 / 4}
$$

where

$$
G=P_{0}(1-S)^{2} \exp (2 g)
$$

is the total gain, and

$$
P_{0}=\cos ^{2}\left(\frac{\pi}{2} \frac{U_{0}}{U_{\lambda / 4}}\right),
$$

after some transformations we obtain the final formula for laser pulse duration:

$$
\tau_{L M}=\frac{\tau_{L} \tau_{M}}{\sqrt[4]{\tau_{M}^{4}+\tau_{L}^{4}}}
$$

Formulas (12)-(15) specify the set of parameters that determine the pulse duration $\tau_{L M}$. These include the characteristics of the active media, RF modulator, laser resonator and the feedback control circuit. In the case of strong modulation the most influential parameters are amplitude voltage and the gain linewidth, as $\tau_{L M}$ is inversely proportional to their square root. The pulse duration $\tau_{L M}$ is proportional to the square root of RF signal period, while the other factors are slowly varying functions. 
Without the RF modulation, the time interval $\Delta T$ between the EOM transmission maximum and the photocurrent maximum was [26]:

$$
\Delta T_{L}=\frac{\tau_{F}}{2 \sqrt{\ln 2}} \sqrt{\ln \left(\frac{2 \sqrt{\ln 2} R C}{\tau_{F}} \frac{\left(\exp \left(T_{r} /(m R C)\right)-1\right)}{\sqrt{\pi}}\right)} .
$$

The delay time for the maximum transmission case with $m$ pulses in the laser cavity is

$$
T_{d_{L}}=(1-1 / m) T_{r}+\frac{\tau_{F}}{2 \sqrt{\ln 2}} \sqrt{\ln \left(\frac{2 \sqrt{\ln 2} R C}{\tau_{F}} \frac{\left(\exp \left(T_{r} /(m R C)\right)-1\right)}{\sqrt{\pi}}\right)} .
$$

Laser pulse duration $\tau_{L M}$ versus $G$ in the SSHML regime with modulation for $m=2$ pulses per round-trip time is presented in Figure 5 for a set of initial voltage $U_{0}$ and modulation depth $a$ values. In the absence of modulation, the minimal laser pulse duration is predicted for $U_{0}=0$. With weak modulation, the laser pulse duration decreases with $G$. For $a>5.25 \%$, the dependence flips to the opposite: $\tau_{L M}$ grows with $G$. The minimal values of laser pulse durations are predicted for $a=150 \%$.
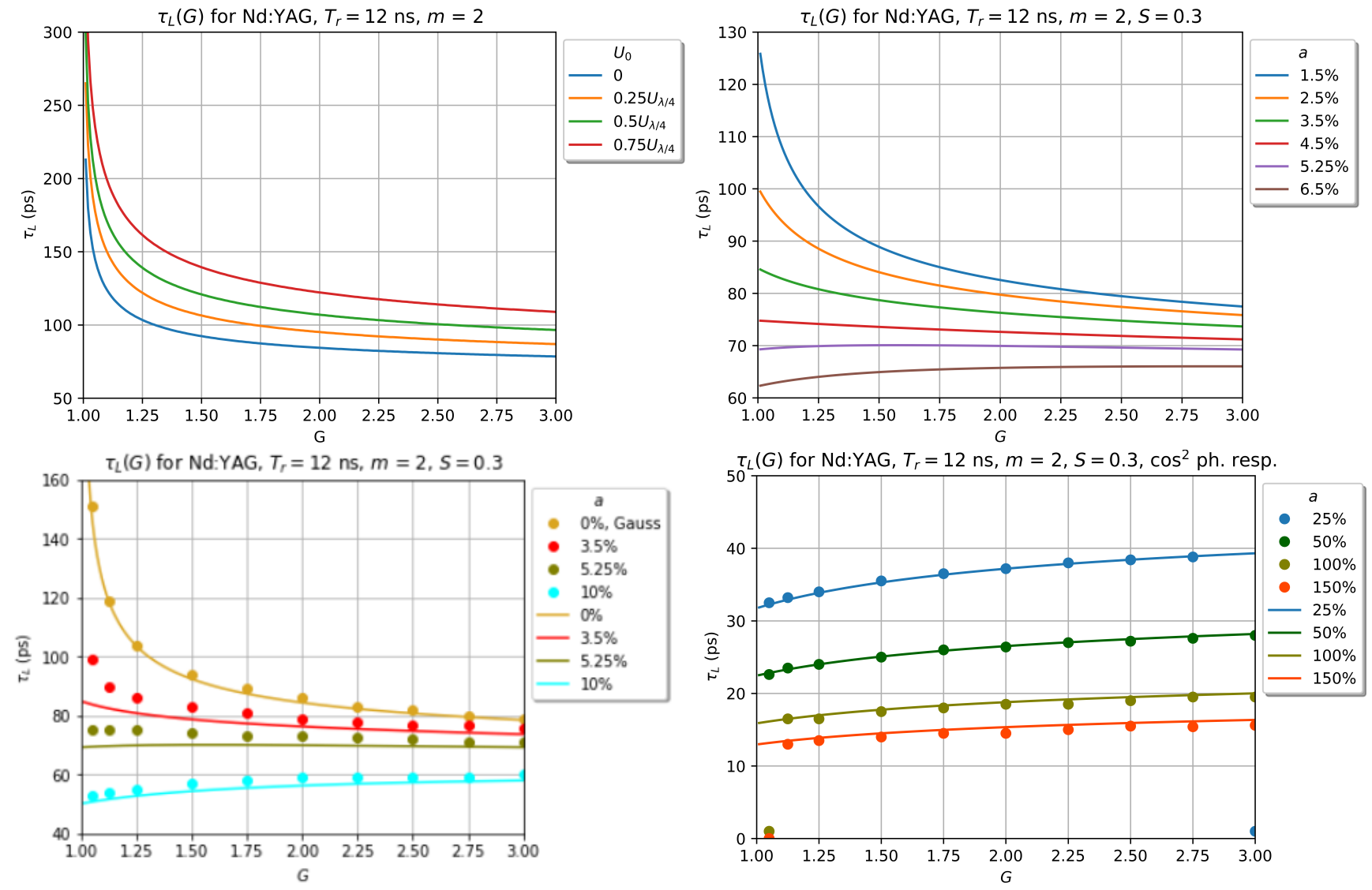

Figure 5. Laser pulse durations $\tau_{L}$ and $\tau_{L M}$ versus $G$ in the SSHML regime with modulation and with $m=2$ pulses per round-trip time $T_{r}=12 \mathrm{~ns}$ : formula (solid curves) and numerical simulation (points) (color online).

Pulse duration values $\tau_{L M}$ estimated for typical $\mathrm{Nd}$ - and $\mathrm{Yb}$-based active media with modulation depth $a=25 \%, 50 \%, 100 \%, 150 \%$ are presented in Tables 1 and 2 . 
Table 1. Pulse duration $\tau_{L M}$ estimated for typical Nd-based active media [32] with modulation depth $a=25 \%, 50 \%, 100 \%, 150 \% . \lambda$ is wavelength, $\sigma_{e}$ is emission cross-section, and $\Delta v$ is emission bandwidth. $G=1.5, S=0.3$.

\begin{tabular}{|c|c|c|c|c|c|c|c|c|}
\hline \multirow{2}{*}{ Active Media } & & \multirow{2}{*}{$\lambda, \mathrm{nm}$} & \multirow{2}{*}{$\sigma_{e}, 10^{-20} \mathrm{~cm}^{2}$} & \multirow{2}{*}{$\Delta v, \mathrm{THz}$} & \multicolumn{4}{|c|}{$\tau_{L M}, \mathrm{ps}$} \\
\hline & & & & & $1 \mathrm{GHz}$ & $3 \mathrm{GHz}$ & $5 \mathrm{GHz}$ & $10 \mathrm{GHz}$ \\
\hline Nd:YAG & & 1064.1 & 88 & 0.12 & $\begin{array}{l}71,54 \\
39,32\end{array}$ & $\begin{array}{l}45,32 \\
23,19\end{array}$ & $\begin{array}{c}35,25 \\
18,14\end{array}$ & $\begin{array}{c}25,18 \\
13,10\end{array}$ \\
\hline \multirow[t]{2}{*}{ Nd:YLF } & $\mathrm{E} \perp \mathrm{c}$ & 1053 & 12 & 0.42 & $\begin{array}{l}38,29 \\
21,17\end{array}$ & $\begin{array}{c}24,17 \\
12,10\end{array}$ & $\begin{array}{c}19,13 \\
9.5,7.7\end{array}$ & $\begin{array}{l}13,9.5 \\
6.7,5.5\end{array}$ \\
\hline & $\mathrm{E} \| \mathrm{c}$ & 1047 & 18 & 0.48 & $\begin{array}{l}36,27 \\
20,16\end{array}$ & $\begin{array}{l}23,16 \\
11,9.4\end{array}$ & $\begin{array}{l}18,13 \\
8.9,7.2\end{array}$ & $\begin{array}{l}13,8.9 \\
6.3,5.1\end{array}$ \\
\hline Nd:YAP & & 1079.5 & 37 & 0.60 & $\begin{array}{c}32,24 \\
18,14\end{array}$ & $\begin{array}{l}20,14 \\
10,8.4\end{array}$ & $\begin{array}{l}16,11 \\
7.9,6.5\end{array}$ & $\begin{array}{l}11,7.9 \\
5.6, \underline{4.6}\end{array}$ \\
\hline \multirow[t]{2}{*}{ Nd:BEL } & $E \| X$ & 1069.8 & 15 & 0.90 & $\begin{array}{l}26,20 \\
14,12\end{array}$ & $\begin{array}{c}16,12 \\
8.4,6.8\end{array}$ & $\begin{array}{l}13,9.2 \\
6.5,5.3\end{array}$ & $\begin{array}{l}9.2,6.5 \\
\underline{4.6}, \underline{3.7}\end{array}$ \\
\hline & $\mathrm{E} \| \mathrm{Y}$ & 1079 & 10 & 3.00 & $\begin{array}{c}14,11 \\
7.9,6.5\end{array}$ & $\begin{array}{l}9.0,6.5 \\
\underline{4.6}, \underline{3.7} \\
\end{array}$ & $\begin{array}{l}7.1, \underline{5.0} \\
3.6, \underline{2.9}\end{array}$ & $\underline{5.0}, \underline{3.5}, \underline{2.1}$ \\
\hline \multirow[t]{2}{*}{ Nd glass } & LG-760 & 1054 & 4.3 & 5.28 & $\begin{array}{l}11,8.2 \\
5.9, \underline{4.9}\end{array}$ & $\begin{array}{l}6.8, \underline{4.9} \\
\underline{3.5}, \underline{2.8}\end{array}$ & $\begin{array}{l}5.3, \underline{3.8} \\
2.7,2.2\end{array}$ & $\begin{array}{l}\underline{3.8}, \underline{2.7} \\
\underline{1.9}, \underline{1.5}\end{array}$ \\
\hline & LG-680 & 1061 & 2.7 & 7.41 & $\begin{array}{l}9.1,6.9 \\
5.0,4.1\end{array}$ & $\begin{array}{l}5.7, \underline{4.1} \\
2.9 \\
\underline{2.4}\end{array}$ & $\frac{4.5}{2.3}, \underline{3.2}$ & $\frac{3.2}{1.6}, \underline{2.3}$, \\
\hline
\end{tabular}

Table 2. Pulse duration $\tau_{L M}$ estimated for typical $\mathrm{Yb}$-based active media [33] with modulation depth $a=25 \%, 50 \%, 100 \%, 150 \%$. $\lambda$ is wavelength and $\Delta v$ is emission bandwidth. $G=1.3, S=0.8$.

\begin{tabular}{|c|c|c|c|c|c|c|c|}
\hline \multirow{2}{*}{ Active Media } & \multirow{2}{*}{$\lambda, \mathrm{nm}$} & \multirow{2}{*}{$\sigma_{e}, 10^{-20} \mathrm{~cm}^{2}$} & \multirow{2}{*}{$\Delta v, \mathrm{THz}$} & \multicolumn{4}{|c|}{$\tau_{L M}, \mathrm{ps}$} \\
\hline & & & & $1 \mathrm{GHz}$ & $3 \mathrm{GHz}$ & $5 \mathrm{GHz}$ & $10 \mathrm{GHz}$ \\
\hline Yb:YAG & 1031 & 2.1 & 2.5 & $\begin{array}{l}21,16 \\
11,9.4\end{array}$ & $\begin{array}{l}13,9.4 \\
6.7,5.4 \\
\end{array}$ & $\begin{array}{l}10,7.3 \\
5.2, \underline{4.2} \\
\end{array}$ & $\begin{array}{l}7.3,5.2, \\
\underline{3.7}, \underline{3.0} \\
\end{array}$ \\
\hline Yb:KGW & 1023 & 2.8 & 7.2 & $\begin{array}{l}13,9.4 \\
6.8,5.5 \\
\end{array}$ & $\begin{array}{l}7.8,5.5 \\
\underline{3.9}, \underline{3.2} \\
\end{array}$ & $\begin{array}{l}6.1, \underline{4.3} \\
\underline{3.0}, \underline{2.5} \\
\end{array}$ & $\underline{4.3}, \underline{3.0}, \underline{1.8}$ \\
\hline $\mathrm{Yb}: \mathrm{CaF}_{2}$ & 1047 & 0.25 & 8.2 & $\begin{array}{l}12,8.8 \\
6.3,5.2 \\
\end{array}$ & $\begin{array}{l}7.3,5.2, \\
\underline{3.7}, \underline{3.0} \\
\end{array}$ & $\begin{array}{l}5.7, \underline{4.0} \\
\underline{2.9}, \underline{2.3} \\
\end{array}$ & $\underline{4.0}, \underline{2.9}, \underline{1.0}, \underline{1.6}$ \\
\hline Yb:glass & 1020 & 0.05 & 10 & $\begin{array}{l}11,8.0 \\
5.7, \underline{4.7} \\
\end{array}$ & $\begin{array}{l}6.6, \underline{4.7} \\
\underline{3.3}, \underline{2.7} \\
\end{array}$ & $\begin{array}{l}5.1, \underline{3.7} \\
\underline{2.6}, \underline{2.1} \\
\end{array}$ & $\underline{3.7}, \underline{2.6}$, \\
\hline Yb:BOYS & 1025 & 0.3 & 17 & $\begin{array}{l}8.2,6.1, \\
\underline{4.4}, \underline{3.6} \\
\end{array}$ & $\begin{array}{l}5.1, \underline{3.6} \\
\underline{2.6}, \underline{2.1} \\
\end{array}$ & $\begin{array}{l}\underline{3.9}, \underline{2.8} \\
\underline{2.0}, \underline{1.6} \\
\end{array}$ & $\begin{array}{l}\underline{2.8}, \underline{2.0} \\
\underline{1.4}, \underline{1.1}\end{array}$ \\
\hline Yb:CALGO & 1050 & 0.8 & 22 & $\begin{array}{c}7.2,5.4 \\
3.9, \\
\end{array}$ & $\frac{4.5}{2.2}, \underline{3.2}$ & $\frac{3.5}{1.7}, \underline{2.5}$, & $\underline{2.5}, \underline{1.7}$ \\
\hline
\end{tabular}

\section{Numerical Simulation Results}

Numerical simulation procedure allows us to investigate the laser operation from the self-start and remains valid for pump power levels sufficient for nonlinear dynamics manifestation. To study the possibility of locking the laser directly to the high-stable RF oscillator, numerical simulation of the laser operation was performed up to the frequency of $10 \mathrm{GHz}$.

The dynamics of laser in the regime of RF modulation was simulated using the following parameters: $0.1 \mathrm{ps}$ time resolution, total calculation time of $2 \times 10^{5} T_{r}$, modulation period $T_{r} / 60$ (corresponds to the frequency of $5 \mathrm{GHz}$ ), constant gain $50 \%$ above threshold $(G=1.5)$. Other parameters are listed in Table 3. Numerical simulation showed that the 
steady-state pulse duration is in a good agreement with the analytical estimates even for the case of weak modulation and excellent for the strong one (as shown in Figure 5). This holds true for all $m=2, \ldots 12$.

Table 3. Numerical simulation parameters.

\begin{tabular}{ccc}
\hline Parameter & Notation & Value \\
\hline Laser cavity round-trip time & $T_{r}$ & $12 \mathrm{~ns}$ \\
Active media & & $\mathrm{Nd}: \mathrm{YG}$ \\
Emission bandwidth & $\Delta v$ & $0.12 \mathrm{THz}$ \\
Beam splitter reflection coef. & $S$ & 0.3 \\
Photodiode response time & $\tau_{F}$ & $500 \mathrm{ps}$ \\
Modulator bias voltage & $U_{0} / U_{\lambda / 4}$ & 0 \\
Total gain & $G$ & 1.5 \\
\hline
\end{tabular}

Table 4 presents the values of laser pulse duration $\tau_{L M}$ at various $m$ and $a$ in the case of Nd:YAG active media. $\tau_{L M}$ decreases with $a$ but is constant over the SSHML intervals of $T_{d}$. The width of the locking intervals decreases with $m$. At $m=12$, the width is only 10 ps which still remains easy to handle experimentally.

Table 4. Laser pulse durations $\tau_{L}$ and $\tau_{L M}$ in the SSHML regime with and without modulation (numerical simulation).

\begin{tabular}{|c|c|c|c|c|c|c|c|}
\hline \multirow{2}{*}{$m$} & \multirow{2}{*}{$R C, \mathrm{ps}$} & \multirow{2}{*}[T_{d_{\operatorname{min}}};T_{d_{\operatorname{max}}}]{, $\mathrm{ps}$} & \multirow{2}{*}{$\tau_{L}, \mathrm{ps}$} & \multicolumn{4}{|c|}{$\tau_{L M}, \mathrm{ps}$, at given $a$} \\
\hline & & & & $25 \%$ & $50 \%$ & $100 \%$ & $150 \%$ \\
\hline 2 & 6000 & {$[6350 ; 6550]$} & 81 & & & & \\
\hline 3 & 4000 & [8330; 8520] & 75 & & & & \\
\hline 4 & 3000 & {$[9340 ; 9520]$} & 75 & & & & \\
\hline 5 & 2400 & {$[10,040 ; 10,080]$} & 79 & 35 & 25 & 17 & 14 \\
\hline 6 & 2000 & {$[10,410 ; 10,470]$} & 75 & & & & \\
\hline 10 & 1200 & {$[11,140 ; 11,180]$} & 68 & & & & \\
\hline 12 & 1000 & {$[11,330 ; 11,340]$} & 68 & & & & \\
\hline
\end{tabular}

Figures 6 and 7 show the evolution of laser radiation fine time structure in the case of $m=12$ without RF modulation and with $a=150 \%$ modulation depth. As can be seen in plots of macroparameters (Figure 8), the variation of $\tau_{L M}$ is smaller with $a=150 \%$ and the build-up time of $\tau_{L M}$ is by an order of magnitude shorter than in the case without RF modulation. The build-up of energy takes comparable times not longer than $10^{4} T_{r}$ in both cases. 
Laser radiation intensity (a.u.) at $a=0 \%$

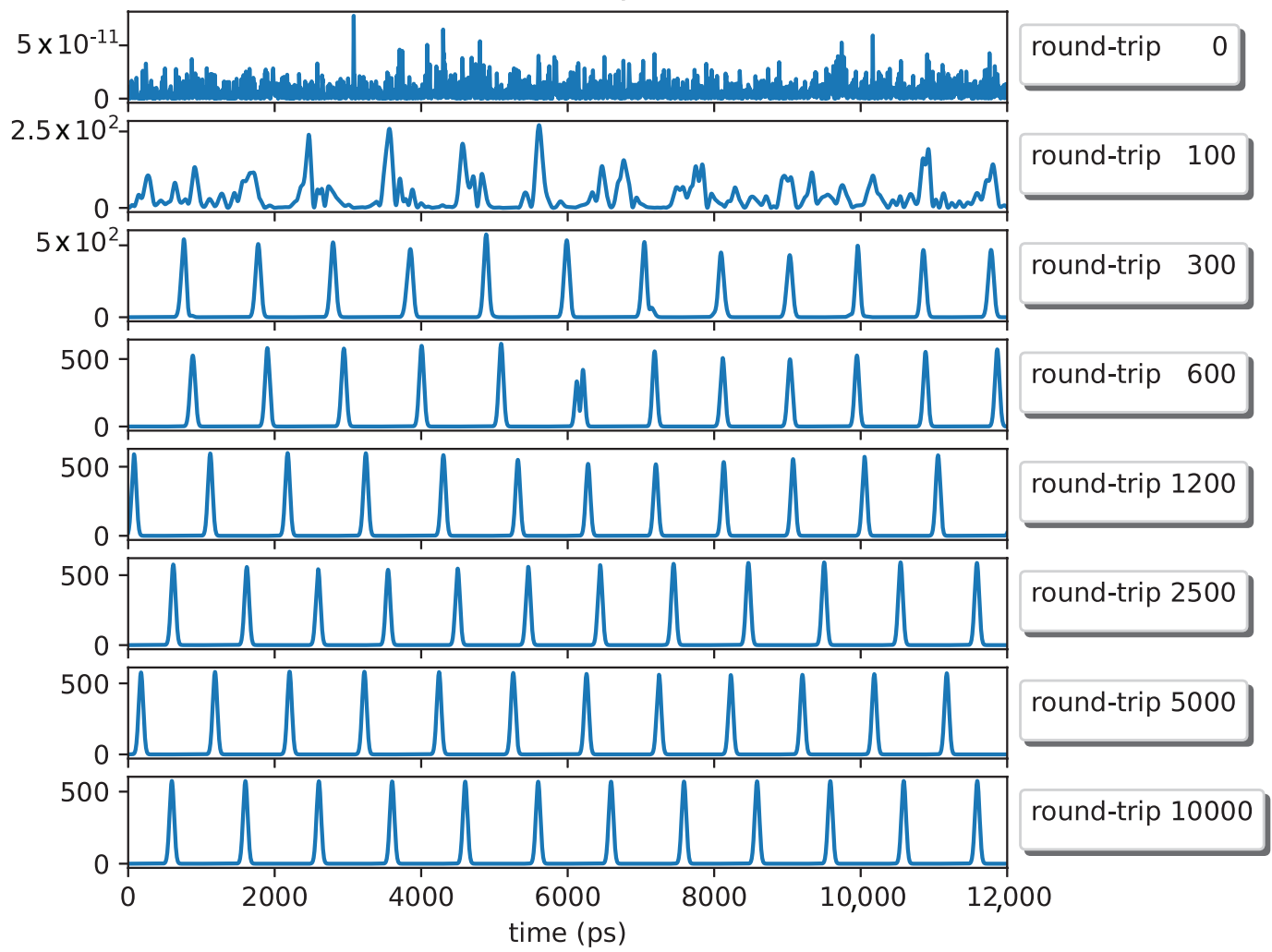

Figure 6. Laser radiation temporal fine structure calculated numerically at modulation depth $a=0 \%$. $T_{d}=11,330 \mathrm{ps}$. Other simulation parameters are given in Table 3 .

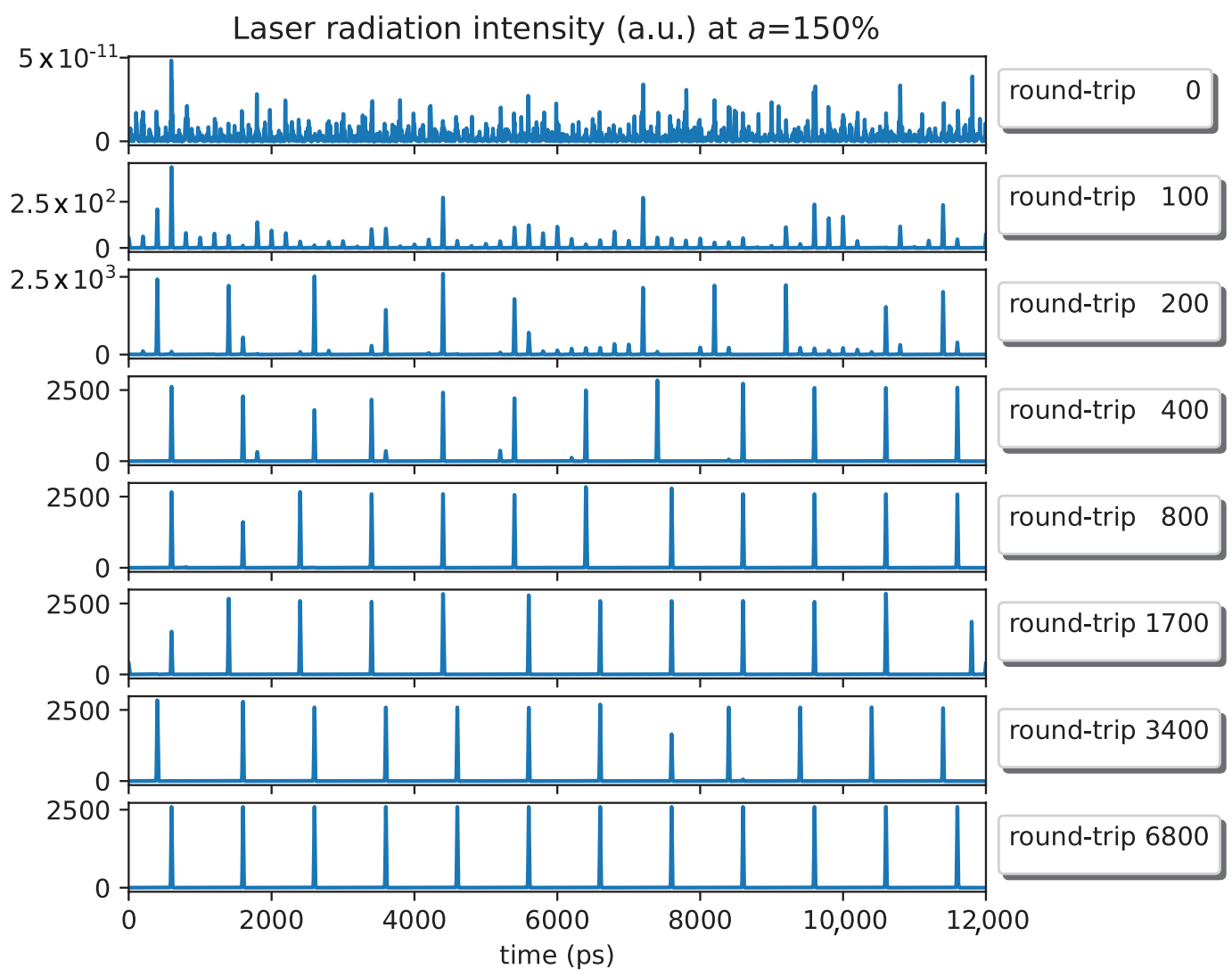

Figure 7. Laser radiation temporal fine structure calculated numerically at modulation depth $a=$ $150 \% . T_{d}=11,330 \mathrm{ps}$. Other simulation parameters are given in Table 3. 

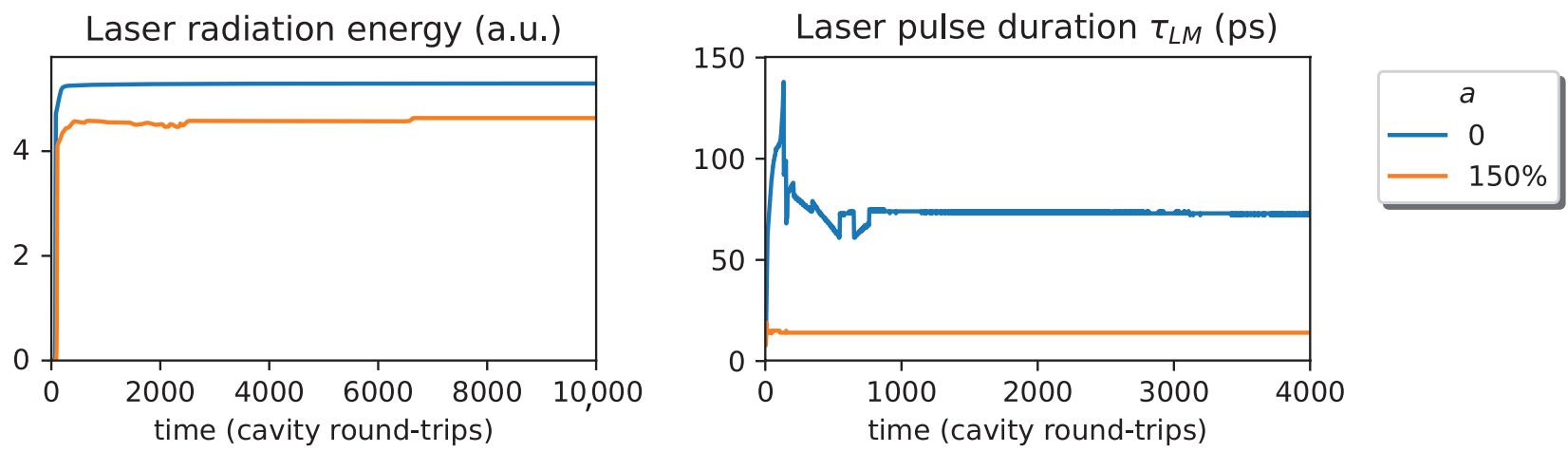

Figure 8. The build-up of laser radiation energy (left) and laser pulse duration (right) at $a=0$ and $150 \% . T_{d}=11,330 \mathrm{ps}$. Other simulation parameters are given in Table 3.

\section{Discussion}

Let us discuss the estimates of $\tau_{L M}$ listed in Tables 1 and 2 where values not greater than $5 \mathrm{ps}$ are underlined as suitable for efficient $X$-ray generation. The threshold value of $\tau_{L M}=5$ ps was selected based on the condition for the maximum accelerator beta-function $\beta$ [34]:

$$
c \tau_{L M}<\beta=\frac{\sigma_{e}^{2} \gamma}{\varepsilon_{0}},
$$

where $c$ is the speed of light, $\gamma$ is the relativistic factor, and $\varepsilon_{0}$ is the normalized emittance of electron bunch. Thus, the selected sets of laser parameters allow highly efficient interaction of light pulse and electron bunch focused by a magnetic lens into a spot with a transverse size $\sigma_{e}$.

Figure 9 presents the dependence of the transverse size on the normalized emittance for the interaction of $1 \mu \mathrm{m}$ radiation and its second harmonic under the conditions for medical range X-ray generation [35]. Based on the data of Table 1 we conclude that the proposed control method is inefficient in L-band accelerators. Lasers based on Nd:YAG also do not satisfy the condition in all bands. Nd:glass lasers are acceptable but do not provide the required repetition rate. Most of the Yb-based media fit the above criterion. However, it is preferable to choose media with larger $\sigma_{e}$ because they provide higher gain levels.

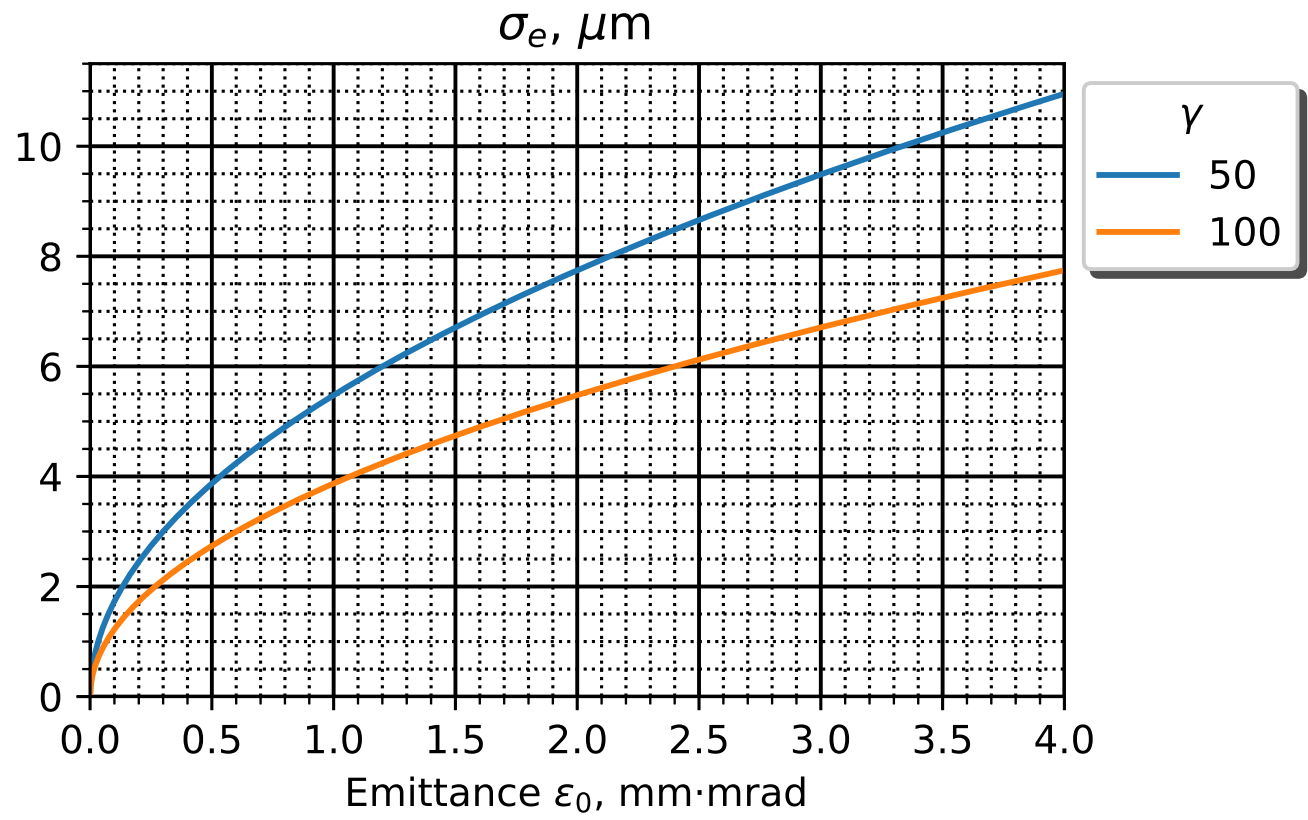

Figure 9. Transverse size of electron bunch in a focal spot $\sigma_{e}$ vs the normalized emittance $\varepsilon_{0}$ at relativistic factor values $\gamma=50$ and 100 . 
Numerical simulation proves that the combined action of RF modulation and SSHML operation is constructive for all $m$ under consideration. With significant amplitude modulation, the laser pulse duration does not depend on $m$ and $T_{d}$ inside each locking interval. Such behaviour differs from previously encountered for (unmodulated) SSHML when the shortest pulse duration occurred at minimum delay time and gradually increased over the rest of the locking delay interval for a certain $m$ [26]. Required precision of $\mathrm{mm}$ level in the delay line, which is rather loose and does not require the application of fine mechanics, is another advantage of the proposed design. Note that the pulse duration without modulation ( $\sim 70-80 \mathrm{ps}$ ) is less than half of the RF signal period. It allows the light pulse to be synchronized with the signal, precisely tied to a single RF pulsation. The necessary condition to implement the proposed concept is the NFB delay in the range of the efficient SSHML in the absence of RF modulation. The proposed control simultaneously shortens the laser pulse and locks the master oscillator to the accelerator. According to the simulation results presented in Table 4 , in the case of Nd:YAG active media, $\tau_{L M}$ drops to $14.0 \mathrm{ps}$ at $150 \%$ modulation depth. Thus it ensures further efficient utilization of laser pulses for bunch generation in photoelectron guns and efficient bunch interaction, e.g., with laser pulses for X-ray generation in Thomson sources.

Numerical modelling made it possible for each value of $m$ to study the manifestations of nonlinear dynamics inherent in delayed NFB control. The limiting value $G_{\text {max }}$ at which the envelope of the pulse train remains unperturbed, monotonically decreases from a value of 3.4 (at $m=2$ ) to 3.0 (at $m=12$ ) when $U_{0}=0$. An increase in $U_{0}$ leads to an expansion of the range of possible gains. Thus, the transition to $U_{0}=0.5$ leads to the transformation of $G_{\text {max }}$ from a value of 6.4 (at $m=2$ ) to 4.4 (at $m=12$ ). The simulation result showed that operational mode at the pre-threshold values makes it possible to expand the range of delays providing for each $m$ the generation of synchronized pulses. In addition, it reduces the build-up time of the SSHML operation. In the case of $\mathrm{Yb}$-based active media, due to the magnitude of the stimulated emission cross-section, we are limited in choosing the $G$ value. For this reason, the SSHML laser pulse duration and output energy for Nd-based active media have lower sensitivity to the gain level than Yb-based active media (see Figure 5 and [26]). Our study shows that high tolerance to the delay mismatch and acceptable buildup time make the laser simple to operate in a pulsed repetitive mode. Thus the proposed laser is an attractive platform for further multi-bunch photoelectron gun development.

The value of $T_{r}=12 \mathrm{~ns}$ is chosen intentionally. In this case, with the $5 \mathrm{GHz}$ oscillator we obtain $k=60$ oscillation maxima per $T_{r}$. Considering the divisors of $k=60$, we come to the conclusion that in such a resonator with the help of a delay in the NFB circuit, it is possible to realize the generation of light pulses synchronized with the RF oscillator with the following periods: $T_{r} / 2, T_{r} / 3, T_{r} / 4, T_{r} / 5, T_{r} / 6, T_{r} / 10, T_{r} / 12, T_{r} / 15, T_{r} / 30, T_{r} / 60$. Thus, it becomes possible to create a research master oscillator for a photoelectron gun with the charge of bunches and their repetition rate varied over a wide range. The transition to a frequency of $10 \mathrm{GHz}$ adds the values $T_{r} / 40$ and $T_{r} / 120$ to the set of possible bunch repetition periods. When the RF oscillator is operating at a frequency of $1 \mathrm{GHz}$, the choice of the repetition period is limited to $T_{r} / 2, T_{r} / 3, T_{r} / 4, T_{r} / 6, T_{r} / 12$. With $3 \mathrm{GHz}$ modulation, the mentioned set of 11 periods requires an increased value of $T_{r}=20 \mathrm{~ns}$. Further advancement of pulse repetition rate into the $\mathrm{GHz}$ range requires a shorter photodiode response time than $\tau_{F}=500$ ps considered in the simulation. Multi-gigahertz pulse repetition rate can be implemented in fiber lasers with low-voltage Mach-Zehnder modulators and ultrafast InGaAs $p-i-n$ photodiodes. In this case, the application of the proposed laser design extends from the accelerator technology into information encryption and other problems of modern photonics.

The advantages of the proposed system compared to other variable repetition-rate oscillators [11,12] are high power and less than 5 ps laser pulse duration. In our design, the intracavity modulator is directly driven by a highly-stable RF oscillator. The proposed system does not include a pulse-picker but is based on feedback control and generates the required number of pulses only due to the choice of feedback delay. The regime of 
harmonic mode-locking is organically tied to the accelerating frequency, and the realizable repetition rates are its subharmonics. Our system is optimal for bulk laser, and the power is not too high to require a stretcher-compressor using fiber amplifiers like in systems with a fiber oscillator or a semiconductor laser. The proposed approach is more suitable for the pulse-repetitive operation.

\title{
7. Conclusions
}

A new concept of a picosecond master oscillator for photoelectron guns suitable for pulse-repetitive operation with variable electron bunch repetition rate was presented. The proposed system generates $m$ equidistant pulses per cavity round-trip time through the electro-optical negative feedback with a variable delay. The pulses are synchronized and shortened using the additional modulator controlled by the RF signal of the second subharmonic of the accelerating voltage. An analytical formula for the short laser pulse duration was derived. In the limit of strong RF modulation, the pulse duration depends on the active media bandwidth, RF modulation depth, and RF frequency. Pulses not longer than 5 ps can be obtained using some broadband $\mathrm{Nd}$ media and all considered $\mathrm{Yb}$ media. Wide stability region, weak sensitivity to gain variation, high tolerance to the feedback delay mismatch and fast build-up make the system suitable for pulsed-repetitive operation in the next-generation X-ray sources.

The resonator configuration with round-trip time $T_{r}$ equal to 60 periods of accelerator field is proposed. In such a laser, by changing the feedback delay time, it is possible to implement the operational mode with the number of pulses: $1 ; 2 ; 3 ; 4 ; 5 ; 6 ; 10 ; 12 ; 15 ; 20 ; 30$ and 60 at the resonator round trip time. With the considered photodiode response time 500 ps, a set of first 8 repetition rates (up to $1 \mathrm{GHz}$ ) is available. It brings the possibility to further optimize the performance of a Laser-Electron X-ray generator. With ultrafast photodiodes, e.g., in fiber systems, the set of laser pulse repetition rates potentially extends to 12 values (up to $10 \mathrm{GHz}$ ) in one configuration of the resonator.

Author Contributions: Conceptualization, M.V.G. and Y.Y.M.; methodology, M.V.G., Y.Y.M. and V.G.T.; software, Y.V.S.; validation, M.V.G., Y.Y.M. and V.G.T.; formal analysis, M.V.G., Y.Y.M. and Y.V.S.; investigation, M.V.G., Y.Y.M. and Y.V.S.; writing-original draft preparation, M.V.G., Y.Y.M. and V.G.T.; writing-review and editing, Y.Y.M. and V.G.T.; visualization, Y.Y.M. All authors have read and agreed to the published version of the manuscript.

Funding: This research was funded by RFBR grant number 19-02-00344-A.

Institutional Review Board Statement: Not applicable.

Informed Consent Statement: Not applicable.

Data Availability Statement: Not applicable.

Acknowledgments: The authors thank I.A. Artyukov and V.I. Shvedunov for fruitful discussions.

Conflicts of Interest: The authors declare no conflict of interest.

\author{
Abbreviations \\ The following abbreviations are used in this manuscript: \\ RF Radio frequency \\ FEL Free-electron laser \\ HML Harmonic mode-locking \\ SSHML Self-starting harmonic mode-locking \\ EOM Electro-optical modulator \\ NFB Negative feedback \\ $T_{r} \quad$ Laser cavity round-trip time \\ ${ }_{L} \quad$ Index $L$ stands for negative-feedback-determined values \\ $\cdot L M \quad$ Index LM means the combined action of NFB and RF modulation
}




\section{Appendix A. Laser Pulse Duration in the Case of SSHML with RF Modulation}

At the first step we obtain the formula for laser pulse duration in the case of arbitrary periodic transmission function. Let a Gaussian pulse circulate in a laser cavity with periodical modulation of losses. The pulse's electrical field can be written as:

$$
E_{K}(t)=A_{K} \exp \left(-\alpha_{K} t^{2}+i \omega_{0} t\right) .
$$

EOM intensity transfer function in the vicinity of its maximum $(t=0)$ can be approximated by Maclaurin series $P(t) \approx P(0)+P^{\prime \prime}(0) t^{2} / 2$, taking into account that $P^{\prime}(0)=0$. Let us denote $P(0)$ by $P_{0}$ and $P^{\prime \prime}(0)$ by $P^{\prime \prime}$. Using $P(t)=\exp \left(\ln (P(t))\right.$ and $\ln (1+x) \approx x-\frac{x^{2}}{2}$ we obtain

$$
P(t) \approx P_{0} \exp \left(\frac{P^{\prime \prime}}{P_{0}}\right) \frac{t^{2}}{2} .
$$

Such a representation allows to work in terms of Gaussian functions to find the solution of Equation (9). The substitution of $P(t), A_{K}$ and $A_{K+1}$ into (9) gives

$$
\begin{gathered}
A_{K+1} \exp \left(-\alpha_{K+1} t^{2}\right)=(1-S) \sqrt{P_{0}} \frac{\exp g}{2 \sqrt{\pi} \tau_{a} \sqrt{g}} \cdot \exp \left(\frac{P^{\prime \prime}}{P_{0}} \frac{t^{2}}{4}\right) . \\
\cdot \int_{-\infty}^{\infty} A_{K} \exp \left(-\alpha_{K} t^{\prime 2}\right) \exp \left(-\left(\frac{t-t^{\prime}-\tau_{a} g}{2 \tau_{a} \sqrt{g}}\right)^{2}\right) d t^{\prime}
\end{gathered}
$$

Using the well-known formula for convolution of two Gaussian functions

$$
\operatorname{conv}\left(e^{-a_{1} t^{2}}, e^{-a_{2} t^{2}}\right)=\frac{\sqrt{\pi} \exp \left(-\frac{a_{1} a_{2} t^{2}}{a_{1}+a_{2}}\right)}{\sqrt{a_{1}+a_{2}}},
$$

we obtain the relations that include amplitudes and pulse duration:

$$
A_{K+1}=A_{K}(1-S) \sqrt{P_{0}} \frac{\exp g}{2 \sqrt{\pi} \tau_{a} \sqrt{g}} \frac{2 \sqrt{\pi} \exp \left(-\frac{\alpha_{K} t^{2}}{4 \alpha_{K} \tau_{a}^{2}+1}\right)}{\sqrt{4 \alpha_{K}+\frac{1}{g \tau_{a}{ }^{2}}}}
$$

and

$$
\exp \left(-\alpha_{K+1} t^{2}\right)=\exp \left(\frac{P^{\prime \prime}}{P_{0}} \frac{t^{2}}{4}\right) \exp \left(-\frac{\alpha_{K} t^{2}}{4 \alpha_{K} g \tau_{a}^{2}+1}\right),
$$

which in steady-state conditions $\left(A_{k+1}=A_{k}, \alpha_{K+1}=\alpha_{K}\right)$ gives

$$
A_{K+1}=A_{K}(1-S) \sqrt{P_{0}} \frac{\exp g}{\tau_{a} \sqrt{g}} \frac{1}{\sqrt{4 \alpha_{K}+\frac{1}{g \tau_{a}^{2}}}}
$$

and

$$
\alpha_{K}=-\frac{P^{\prime \prime}}{P_{0}}+\frac{\alpha_{K}}{4 \alpha_{K} g \tau_{a}^{2}+1} .
$$

Using the relation $\alpha_{K} \tau_{a}^{2} \ll 1$, we obtain

$$
\alpha_{K}=\sqrt{-\frac{P^{\prime \prime}}{16 P_{0} \tau_{a}^{2} g}} .
$$

Pulse duration in intensity representation is

$$
\tau_{L}=\sqrt{\frac{2 \ln 2}{\alpha_{K}}}=\sqrt{8 \ln 2} \sqrt[4]{-\frac{P_{0} \tau_{a}^{2} g}{P^{\prime \prime}}}=\sqrt{\frac{8 \ln 2}{\pi \Delta v}} \sqrt[4]{-g \frac{P_{0}}{P^{\prime \prime}}} .
$$


Now we consider the combined action of NFB and RF modulation. In this case, the transmission function is

$$
P(t)=P_{1}(t) P_{2}(t),
$$

where $P_{1}(t)=\cos ^{2}\left(\frac{\pi}{2} \cdot \frac{U(t)+U_{0}}{U_{\lambda / 4}}\right)$ and $P_{2}(t)=\cos ^{2}\left(\frac{\pi}{2} \frac{U_{m}}{U_{\lambda / 4}} \cdot \sin \left(\frac{2 \pi}{T} t\right)\right)$.

$$
\begin{gathered}
P^{\prime}=P_{1}^{\prime}(t) P_{2}(t)+P_{1}(t) P_{2}^{\prime}(t), \\
P^{\prime \prime}(t)=P_{1}^{\prime \prime}(t) P_{2}(t)+2 P_{1}^{\prime}(t) P_{2}^{\prime}(t)+P_{1}(t) P_{2}^{\prime \prime}(t) .
\end{gathered}
$$

In maximum

$$
\begin{gathered}
\tau_{L M}=\sqrt{\frac{8 \ln 2}{\pi \Delta v}} \sqrt[4]{\frac{-g P_{1_{\max }}}{P^{\prime \prime}+P_{1_{\max }} P_{2}^{\prime \prime}}} \\
P_{2}^{\prime}(t)=-\sin \left[\pi \frac{U_{0}}{U_{\lambda / 4}} \sin \frac{2 \pi}{T} t\right] \frac{\pi^{2}}{T} \frac{U_{0}}{U_{\lambda / 4}} \cos \frac{2 \pi}{T} t \\
P_{2}^{\prime \prime}(t)=-\cos \left[\pi \frac{U_{0}}{U_{\lambda / 4}} \sin \frac{2 \pi}{T} t\right] \frac{\pi^{4}}{T^{2}}\left(\frac{U_{0}}{U_{\lambda / 4}}\right)^{2} \cdot 2 \cos \frac{2 \pi}{T} t .
\end{gathered}
$$

In maximum

$$
P_{2}^{\prime \prime}(t)=-\frac{2 \pi^{4}}{T^{2}}\left(\frac{U_{0}}{U_{\lambda / 4}}\right)^{2} .
$$

Modulation-determined duration is

$$
\begin{gathered}
\tau_{M}=\sqrt{\frac{8 \ln 2}{\pi \Delta v}} \sqrt[4]{\frac{-g}{P_{2}^{\prime \prime}}} \\
\tau_{M}=\sqrt{\frac{8 \ln 2}{\pi \Delta v}} \sqrt[4]{\frac{g T^{2}}{2 \pi^{4}}\left(\frac{U_{\lambda / 4}}{U_{0}}\right)^{2}} \\
\tau_{M}=\frac{2}{\pi} \sqrt{\frac{\ln 2}{\pi}}\left(\frac{T}{\Delta v} \cdot \frac{U_{\lambda / 4}}{U_{m}}\right)^{1 / 2}\left(\ln \frac{G}{P_{0}(1-S)^{2}}\right)^{1 / 4}
\end{gathered}
$$

where

$$
P_{0}=\cos ^{2} \frac{\pi U_{0}}{2 U_{\lambda / 4}}
$$

and

$$
g=\frac{1}{2} \ln \frac{G}{P_{0}(1-S)^{2}} .
$$

After some transformations we obtain the final formula for laser pulse duration:

$$
\tau_{L M}=\frac{\tau_{L} \tau_{M}}{\sqrt[4]{\tau_{M}^{4}+\tau_{L}^{4}}}
$$

where $\tau_{L}$ is the laser pulse duration in the maximum transmission case without modulation (as derived in [26]):

$$
\begin{gathered}
\tau_{L}=\left(\frac{2 \sqrt{\ln 2}}{\pi}\right)^{3 / 4} \frac{\left(\tau_{F} \cdot R C\right)^{1 / 4}}{\Delta v_{L}^{1 / 2}}\left(\ln \frac{G}{P_{0}(1-S)^{2}}\right)^{\frac{1}{4}} . \\
\cdot\left[\left(\frac{2}{\pi} \arccos \sqrt{\frac{P_{0}}{G}}-\frac{U_{0}}{U_{\lambda / 4}}\right) \sqrt{\frac{G}{P_{0}}-1}\right]^{-\frac{1}{4}}\left[\ln \left(\frac{2 \sqrt{\ln 2}}{\sqrt{\pi}}\left(\exp \frac{T_{r}}{m R C}-1\right) \frac{R C}{\tau_{F}}\right)\right]^{-\frac{1}{8}} .
\end{gathered}
$$




\section{References}

1. Kerse, C.; Kalaycioglu, H.; Elahi, P.; Akcaalan, O.; Ilday, F.O. 3.5-ghz intra-burst repetition rate ultrafast yb-doped fiber laser. Opt. Comm. 2016, 1, 404-409. [CrossRef]

2. Soibel, A.; Wright, M.; Farr, W.; Keo, S.; Hill, C.; Yang, R.Q.; Liu, H.C. Mid-infrared interband cascade lasers for free-space laser communication. In Proceedings of the Proceedings Volume 7199, Free-Space Laser Communication Technologies XXI, 71990E (2009), San Jose, CA, USA, 24-29 January 2009.

3. Delfyett, P.; Christopher, D.; Yilmaz, T. Ultralow Noise Optical Clock for High Speed Sampling Aplications. U.S. Patent 2004/6735229 B1, 11 May 2004.

4. Priebe, G.; Laundy, D.; Phillips, P.J.; Graham, D.M.; Jamison, S.P.; Vassilev, S.; Seddon, E.A.; Rosenzweig, J.B.; Krafft, G.A.; Heinzl, T.; et al. First results from the daresbury compton backscattering X-ray source (cobald). In Proceedings of the Proceedings Volume 7805, Hard X-ray, Gamma-Ray, and Neutron Detector Physics XII, 780513 (2010), San Diego, CA, USA, 1-5 August 2010.

5. Will, I.; Templin, H.I.; Schreiber, S.; Sandner, W. Photoinjector drive laser of the flash FEL. Opt. Express 2011, 19, $23770-23781$. [CrossRef] [PubMed]

6. Shimizu, H.; Akemoto, M.; Arai, Y.; Araki, S.; Aryshev, A.; Fukuda, M.; Fukuda, S.; Haba, J.; Hara, K.; Hayano, H.; et al. X-ray generation by inverse compton scattering at the superconducting rf test facility. Nucl. Instrum. Methods Phys. Res. Sect. A Accel. Spectrom. Detect. Assoc. Equip. 2015, 772, 26-33. [CrossRef]

7. Terunuma, N.; Murata, A.; Fukuda, M.; Hirano, K.; Kamiya, Y.; Kii, T.; Kuriki, M.; Kuroda, R.; Ohgaki, H.; Sakaue, K.; et al Improvement of an s-band rf gun with a cs2te photocathode for the kek-atf. Nucl. Instrum. Methods Phys. Res. Sect. A Accel. Spectrom. Detect. Assoc. Equip. 2010, 613, 1-8. [CrossRef]

8. Bessonov, E.G.; Gorbunkov, M.V.; Ishkhanov, B.S.; Kostryukov, P.V.; Maslova, Y.Y.; Shvedunov, V.I.; Tunkin, V.G.; Vinogradov, A.V. Laser-electron generator for x-ray applications in science and technology. Laser Part. Beams 2008, 26, 489-495. [CrossRef]

9. Veisman, M.K.; Andreev, N.E. Dependence of emittance on the length of the electron bunch during laser- plasma acceleration in guiding structures. Kvantovaja Elektron. 2020, 50, 392-400. [CrossRef]

10. Krasilnikov, M.; Stephan, F.; Asova, G.; Grabosch, H.J.; Groß, M.; Hakobyan, L.; Isaev, I.; Ivanisenko, Y.; Jachmann, L.; Khojoyan, M.; et al. Experimentally minimized beam emittance from an L-band photoinjector. Phys. Rev. Spec.-Top.-Accel. Beams 2012, 15, 100701. [CrossRef]

11. Kienle, F.; Chen, K.K.; Alam, S.U.; Gawith, C.B.; Mackenzie, J.I.; Hanna, D.C.; Richardson, D.J.; Shepherd, D.P. High-power, variable repetition rate, picosecond optical parametric oscillator pumped by an amplified gain-switched diode. Opt. Express $\mathbf{2 0 1 0}$ 18, 7602-7610. [CrossRef]

12. Fu, Q.; Wu, Y.; Liang, S.; Shardlow, P.C.; Shepherd, D.P.; Alam, S.U.; Xu, L.; Richardson, D.J. Controllable duration and repetition-rate picosecond pulses from a high-average-power OP-GaAs OPO. Opt. Express 2020, 28, 32540-32548. [CrossRef]

13. Becker, M.; Kuizenga, D.; Siegman, A. Harmonic mode locking of the Nd:YAG laser. IEEE J. Quantum Electron. 1972, 8, 687-693. [CrossRef]

14. Koo, J.; Park, J.; Lee, J.; Jhon, Y.M.; Lee, J.H. Femtosecond harmonic mode-locking of a fiber laser at 3.27 GHz using a bulk-like, MoSe2 based saturable absorber. Opt. Express 2016, 24, 10575-10589. [CrossRef] [PubMed]

15. Lecaplain, C.; Grelu, P. Multi-gigahertz repetition-rate-selectable passive harmonic mode locking of a fiber laser. Opt. Exp. 2013, 21, 10897-10902. [CrossRef] [PubMed]

16. Hao, Q.; Wang, Y.; Luo, P.; Hu, H.; Zeng, H. Self-starting dropout-free harmonic mode-locked soliton fiber laser with a low timing jitter. Opt. Lett. 2017, 42, 2330-2333. [CrossRef] [PubMed]

17. Peng, J.; Zhan, L.; Luo, S.; Shen, Q. Passive harmonic mode-locking of dissipative solitons in a normal-dispersion Er-doped fiber laser. J. Lightwave Technol. 2013, 31, 3009-3014. [CrossRef]

18. Mao, Y.; Tong, X.; Wang, Z.; Zhan, L.; Hu, P.; Chen, L. Wavelength tunable $10 \mathrm{GHz}$ actively harmonic mode-locked fiber laser based on semiconductor optical amplifier. App. Phys. B 2015, 121, 517-521. [CrossRef]

19. Li, W.; Yin, Z.; Qiu, J.; Wu, J.; Lin, J. Tunable active harmonic mode-locking Yb-doped fiber laser with all-normal dispersion. IEEE Phot. Techn. Lett. 2013, 25, 2247-2250. [CrossRef]

20. Gorbunkov, M.V.; Konyashkin, A.V.; Kostryukov, P.V.; Morozov, V.B.; Olenin, A.N.; Rusov, V.A.; Telegin, L.S.; Tunkin, V.G.; Shabalin, Y.V.; Yakovlev, D.V. Pulsed-diode-pumped, all-solid-state, electro-optically controlled picosecond Nd:YAG lasers. Quantum Electron. 2005, 35, 2. [CrossRef]

21. Scott, R.P.; Bennet, C.V.; Kolner, B.H. AM and high-harmonic FM laser mode-locking. Appl. Opt. 1997, 36, 5908-5912. [CrossRef]

22. Godil, A.A.; Hou, A.S.; Auld, B.A.; Bloom, D.M. Harmonic mode-locking of a Nd:BEL laser using a 20-GHz dielectric resonator/optical modulator. Opt. Lett. 1991, 16, 1765-1767. [CrossRef]

23. Longhi, S.; Laporta, P.; Taccheo, S.; Svelto, O. Third-order-harmonic mode-locking of a bulk erbium:ytterbium:glass laser at a $2.5 \mathrm{GHz}$ repetition rate. Opt. Lett. 1994, 19, 1985-1987. [CrossRef]

24. Hughes, D.W.; Barr, J.R.M.; Hanna, D.C. Mode locking of a diode-laser-pumped Nd:glass laser by frequency modulation. Opt. Lett. 1991, 16, 147-149. [CrossRef] [PubMed]

25. Gorbunkov, M.V.; Maslova, Y.Y.; Tunkin, V.G.; Shabalin, Y.V.; Yakovlev, D.V. Application of optoelectronic negative feedback to ordering of the temporal structure of the diode-pumped nd:ylf laser radiation. Bull. Lebedev Phys. Inst. 2016, 43, 217-222. [CrossRef] 
26. Gorbunkov, M.V.; Maslova, Y.Y.; Shabalin, Y.V.; Tunkin, V.G. Analysis of Self-Starting Harmonic Mode-Locking in an ElectroOptic-Feedback Laser. IEEE J. Quantum Electron. 2021, 57, 1-8. [CrossRef]

27. Gorbunkov, M.V.; Maslova, Y.Y.; Shabalin, Y.V. On master oscillator of laser-electron x-ray generator. J. Russ. Laser Res. 2019, 40, 547-553. [CrossRef]

28. Gorbunkov, M.; Maslova, Y.; Vinogradov, A. Optical unit of laser-electron x-ray generator designed for medical applications. Nucl. Instrum. Methods Phys. Res. Sect. A Accel. Spectrom. Detect. Assoc. Equip. 2009, 608, S32-S35. [CrossRef]

29. Gorbunkov, M.; Shabalin, Y. Two-loop feedback controlled laser: New possibilities for ultrashort pulses generation and high-level stabilization. In Proceedings of the Proceedings Volume 4751, ICONO 2001: Nonlinear Optical Phenomena and Nonlinear Dynamics of Optical Systems, Minsk, Belarus, 26 June-1 July 2001.

30. Herrmann, J.; Wilhelmi, B. Lasers for Ultrashort Light Pulses; Academie: Berlin, Germany, 1987.

31. Smith, P. Mode-locking of lasers. Proc. IEEE 1970, 58, 1342-1357. [CrossRef]

32. Penzkofer, A. Solid state lasers. Progr. Quant. Electr. 1988, 12, 291-427. [CrossRef]

33. Druon, F.; Boudeile, J.; Zaouter, Y.; Hanna, M.; Balembois, F.; Georges, P.; Petit, J.; Golner, P.; Viana, B. New yb-doped crystals for high-power and ultrashort lasers. In Proceedings of the Proceedings Volume 6400, Femtosecond Phenomena and Nonlinear Optics III, 64000D (2006), Stockholm, Sweden, 11-14 September 2006.

34. Brown, W.J.; Hartemann, F.V. Brightness Optimization of Ultra-Fast Thomson Scattering X-ray Sources. AIP Conf. Proc. 2004, 737, 839-845.

35. Artyukov, I.A.; Bessonov, E.G.; Feshchenko, R.M.; Gorbunkov, M.V.; Maslova, Y.Y.; Popov, N.L.; Dyachkov, N.V.; Postnov, A.A.; Vinogradov, S.L.; Vinogradov, A.V. Design study of Thomson Laser-Electron X-ray Generator (LEX) for Millisecond Angiography. IOP Conf. Ser. J. Phys. Conf. Ser. 2017, 784, 012002. [CrossRef] 